\title{
Incidencia acumulada de insuficiencia renal aguda en el posoperatorio de cirugía cardíaca
}

\author{
Dres. María Victoria Ramos, Marcos Pouso, Jorge Pouso*
}

\begin{abstract}
Resumen
Introducción: la insuficiencia renal aguda (IRA) constituye una causa frecuente de morbimortalidad en el posoperatorio de cirugía cardíaca, con incidencia variable según la definición utilizada y los factores derivados del paciente y del procedimiento quirúrgico.
\end{abstract}

Objetivo: los objetivos de este artículo fueron determinar la incidencia de IRA en un centro de cirugía cardíaca, la presencia de predictores y su asociación con mortalidad quirúrgica.

Métodos: estudio prospectivo y observacional con elementos analíticos. Se incluyeron todos los pacientes con función renal normal y sin antecedentes de enfermedad renal sometidos a cirugía cardíaca en el período julio 2014 - julio 2015 . Se determinó la creatininemia basal y a las 24 y 48 horas de la cirugía. La IRA fue definida utilizando el criterio RIFLE. Se valoró mortalidad quirúrgica y se analizaron variables pre e intraoperatorias como predictoras de desarrollo de IRA utilizando análisis uni y multivariado.

Resultados: cumplieron los criterios de inclusión 400 pacientes. La incidencia de IRA por criterio RIFLE fue 10,3\% (IC95\% 8,7-11,8); la mortalidad quirúrgica en este grupo alcanzó 19,5\%, mientras que en los controles fue 1,9\%. Las variables asociadas al desarrollo de IRA fueron EuroSCORE más elevado, cirugía combinada, uso de circulación extracorpórea (CEC) y clampeo aórtico. La cirugía de revascularización miocárdica (CRM) presentó una asociación inversa con la aparición de IRA y constituyó un predictor independiente en el análisis multivariado. La presencia de IRA fue un predictor independiente de mortalidad quirúrgica.

Conclusiones: la incidencia de IRA es frecuente en el posoperatorio de cirugía cardíaca y se encuentra dentro de los valores internacionales. Su presencia implica mayor mortalidad quirúrgica.

Palabras clave: INSUFICIENCIA RENAL

CIRUGÍA TORÁCICA

PERÍODO POSOPERATORIO

RIFLE

CIRCULACIÓN EXTRACORPÓREA

\section{Accumulated incidence of acute renal failure in postoperatory of cardiac surgery}

\section{Summary}

Introduction: acute renal failure (ARF) is a frequent cause of morbidity and mortality in the postoperative period of cardiac surgery, with variable incidence depending on the definition used, factors derived from the patient and the surgical procedure.

Objective: determine the incidence of ARF in a cardiac surgery center, the presence of predictors and its association with surgical mortality.

Methods: this is a prospective and observational study with analytical elements. All patients with normal renal function and no history of kidney disease undergoing cardiac surgery were included in the period from July 2014 to July 2015. Baseline creatininemia was determined, and 24 and 48 hours later, after surgery. ARF was defined using the RIFLE criterion. Surgical mortality, pre and intra-operative variables were assessed using univariate and multivariate analysis.

Results: four hundred patients met the inclusion criteria. The incidence of acute renal failure by RIFLE criterion was

Centro Cardiovascular Casa de Galicia.

Correspondencia: Dra. María Victoria Ramos. Correo electrónico: mariavictoriaramos@outlook.es

* Fallecido.

Recibido Feb 27, 2018; aceptado Jun 7, 2018 
10,3\% (95\% CI 8,7-11,8), surgical mortality in this group reached 19,5\% while in controls it was 1,9\%. The variables associated with the presence of ARF were the higher EuroSCORE, combined surgery, use of extracorporeal circulation (ECC) and aortic clamping. The myocardial revascularization surgery (MRS) presented an inverse association and was an independent predictor in the multivariate analysis. The presence of ARF was an independent predictor of surgical death.

Conclusions: the incidence of ARF is frequent in postoperatory of cardiac surgery and is within the international values. Its presence implies greater surgical mortality.

Key words:

\section{RENAL INSUFFICIENCY \\ THORACIC SURGERY \\ POSTOPERATIVE PERIOD \\ RIFLE}

EXTRACORPOREAL CIRCULATION

\section{Introducción}

La injuria, insuficiencia o falla renal aguda (IRA) como morbilidad en el posoperatorio de cirugía cardíaca (POCC) es un conocido factor de mortalidad quirúrgica independiente de otras causas ${ }^{(1-3)}$. Se han publicado trabajos en los que un incremento de solo $0,3 \mathrm{mg} / \mathrm{dl}$ en la creatinina sérica tiene considerable impacto en la supervivencia a 30 días $^{(4)}$.

$\mathrm{Su}$ incidencia es variable en los distintos estudios, oscilando entre $5 \%$ y $20 \%{ }^{(5-7)} ; 1 \%$ a $2 \%$ de los pacientes requerirán diálisis ${ }^{(6,8)}$.

En nuestro centro, en el período comprendido entre 1995 y 2013, de 7.873 pacientes intervenidos sin enfermedad renal crónica (ERC) previa, 700 desarrollaron IRA según la definición de la Sociedad de Cirujanos Torácicos (STS por su sigla en inglés), que considera creatininemia $\geq 2,0 \mathrm{mg} / \mathrm{dl}$, (incidencia acumulada 8,9\% [IC95\% 8,3-9,5\%]). Se han desarrollado varios criterios para definir insuficiencia renal. Entre ellos, destacan además de STS: RIFLE y AKIN.

Los factores de riesgo que deben tenerse en cuenta son: el tipo de cirugía, sabiendo que los pacientes que se someten a operación combinada [cirugía de revascularización miocárdica (CRM) y valvular] tienen una incidencia superior cuando se la compara con procedimientos aislados ${ }^{(9-11)}$, y el uso de circulación extracorpórea (CEC), clampeo aórtico y su duración, ya que algunos datos aportados por la bibliografía demuestran mayor incidencia en los pacientes con CEC que en los pacientes sin $\operatorname{CEC}^{(6,9,12,-14)}$.

El desarrollo de IRA, a su vez, se asocia a complicaciones infecciosas de gravedad variable, incremento en la estadía hospitalaria y mal pronóstico a

Principales aportes

- El artículo permite conocer la incidencia de insuficiencia renal aguda y su influencia en la mortalidad quirúrgica en la población estudiada, aportando datos nacionales.

- Compara distintos criterios para definir insuficiencia renal aguda. largo plazo con aumento significativo de la mortali$\operatorname{dad}^{(1,4,6,9,15,16)}$. Considerando todo lo previamente expuesto, los objetivos del trabajo fueron determinar la incidencia acumulada de IRA en el POCC en nuestro servicio, evaluar la existencia de predictores de IRA y mortalidad asociada. Para definir IRA se utilizó el consenso RIFLE basado en la creatininemia $^{(17)}$. Se comparó la incidencia utilizando la definición RIFLE vs STS.

\section{Métodos}

Estudio prospectivo observacional con elementos analíticos. Un total de 522 pacientes mayores de 18 años fueron admitidos en el servicio de cirugía cardíaca al menos 24 horas antes de someterse a los siguientes procedimientos: cirugía valvular, CRM, procedimientos sobre aorta torácica, corrección de cardiopatía congénita o combinaciones de los anteriores. El período de tiempo considerado fue desde el 16 de julio de 2014 al 15 de julio de 2015. Los pacientes con historia de ERC de cualquier estadio fueron excluidos del estudio. Posteriormente, se realizaron tomas de sangre para determinación de creatininemia basal. Los puntos de corte considerados normales fueron $\leq 1,1 \mathrm{mg} / \mathrm{dl}$ en mujeres $\mathrm{y} \leq 1,3$ $\mathrm{mg} / \mathrm{dl}$ en hombres ${ }^{(18)}$. Los pacientes con valores por encima de los establecidos fueron excluidos del estudio. Los incluidos se sometieron a determinación de creatininemia a las 24 y 48 horas del posoperatorio. Se definió IRA utilizando la clasificación RIFLE mediante el incremento en el valor de creatininemia comparado con el basal.

Se recogieron datos preoperatorios según definición de STS, entre ellos: diabetes mellitus (DM) con o sin requerimiento de insulina, hipertensión arterial (HTA), tabaquismo, dislipemia, presencia de fibrilación auricular crónica (FAC), obesidad, edad y fracción de eyección del ventrículo izquierdo (FEVI). Se consideraron diabéticos aquellos pacientes que recibían insulina o hipoglicemiantes orales, hipertensos los pacientes con presión arterial mayor a 140/90 mmHg, historia de HTA o necesidad de medicación 
Tabla 1. Clasificación RIFLE. Modificado de Alcázar R, et al. Avances en la insuficiencia renal aguda en el año 2008. Nefrol 2009; 29: 82-7.

\begin{tabular}{lll}
\hline Estadio & \multicolumn{1}{c}{ Criterio } & \multicolumn{1}{c}{ Diuresis } \\
\hline Riesgo & Aumento $\mathrm{Cr}>1,5-2$ veces basal & $<0,5 \mathrm{mg} / \mathrm{kg} / \mathrm{h}$ en $6 \mathrm{~h}$ \\
Injuria & Aumento $\mathrm{Cr}>2-3$ veces basal & $<0,5 \mathrm{mg} / \mathrm{kg} / \mathrm{h}$ en $12 \mathrm{~h}$ \\
Falla & Aumento $\mathrm{Cr}>3$ veces basal & $<0,3 \mathrm{mg} / \mathrm{kg} / \mathrm{h}$ en $24 \mathrm{~h}$ o anuria en $12 \mathrm{~h}$ \\
\hline Cr: creatininemia. & & \\
\hline
\end{tabular}

antihipertensiva y dislipémicos quienes tenían historia de hipercolesterolemia o hipertrigliceridemia. Se consideraron obesos aquellos pacientes con índice de masa corporal igual o superior a 30. La FEVI se determinó en el preoperatorio mediante ecocardiograma Doppler transtorácico.

Dentro de las cirugías, se destacó si el paciente cursaba endocarditis infecciosa, uso de balón de contrapulsación intraaórtico (BIAC) y de inotrópicos por más de 48 horas. Se determinó el uso de CEC y clampeo aórtico y los tiempos empleados. Los pacientes que contaran con al menos un valor posoperatorio de creatininemia se consideraron en el estudio, independientemente del desenlace.

Población: fueron seleccionados pacientes consecutivos que ingresaron al servicio para cirugía cardíaca. Se tomaron muestras de sangre para determinación de creatinina sérica basal en el preoperatorio y en el posoperatorio a las 24 y 48 horas, sin solicitar consentimiento a los pacientes, ya que se trata de un procedimiento de rutina en el servicio.

La concentración de creatinina fue definida por la reacción de Jaffe ${ }^{(19)}$. Los valores obtenidos se expresaron en $\mathrm{mg} / \mathrm{dl}$. Los reactivos fueron provistos por Laboratorio Wiener (creatinina cinética AA) y se calibraron mediante calibrador A plus Wiener. El laboratorio de nuestro servicio cuenta con controles internos de calidad (diarios) y controles mensuales externos enviados a RIQAS (Esquema internacional de aseguramiento de la calidad).

Criterios de inclusión: pacientes de cualquier edad y sexo que fueron sometidos a cirugía cardíaca con o sin CEC. Valor de creatininemia basal $\leq 1,1$ $\mathrm{mg} / \mathrm{dl}$ en mujeres $\mathrm{y} \leq 1,3 \mathrm{mg} / \mathrm{dl}$ en hombres ${ }^{(18)}$.

Criterios de exclusión: antecedentes de ERC.

Definiciones: partiendo de pacientes con determinación normal de creatinina sérica en el preoperatorio, fueron considerados como nuevos casos de IRA aquellos con un deterioro en la función renal súbito y sostenido por más de 24 horas en las primeras 48 horas del POCC. Se consideró aumento significativo el mayor a 1,5 veces el valor basal. En la tabla 1 se resume el criterio de clasificación RIFLE. No se consideró el criterio de diuresis, que si bien está incluido en la definición, en el POCC el manejo de fluidos varía ampliamente según las características del paciente y el tipo de cirugía ${ }^{(20)}$.

Mortalidad operatoria: según la definición de STS se consideró cualquier muerte, independientemente de la causa, que ocurriera dentro de los 30 días posteriores a la cirugía dentro o fuera del hospital, o después de 30 días durante la misma hospitalización posterior a la operación ${ }^{(21)}$.

Métodos estadísticos: las variables continuas se expresaron como media y su desviación estándar. Las categóricas se expresaron como porcentaje. La asociación de las variables categóricas con el evento se testeó mediante test exacto de Fisher. Las variables que se asociaron en el univariado luego se utilizaron para el análisis multivariado mediante regresión logística binaria. Para las variables continuas, primero se realizaron pruebas no paramétricas de contraste con la distribución normal (prueba de Kolmogórov-Smirnov para una muestra y test de Lilliefors corregido). En ningún caso la distribución de las variables resultó ser normal. Luego se compararon las distribuciones entre los grupos mediante U de Mann Whitney. Se consideró significativo un valor de $\mathrm{p}<0,05$.

\section{Reclutamiento}

En el período comprendido entre el 16 de julio de 2014 y el 15 de julio de 2015, 522 pacientes se sometieron a cirugía cardíaca. De estos, 7,9\% (41/522 pacientes) era portador de ERC, siendo excluido del estudio. En los 481 pacientes restantes se determinó la creatininemia basal: en tres de ellos $(0,6 \%)$ los datos no provenían de nuestro laboratorio, por lo que también se excluyeron. El 16,2\% (78/481 pacientes) presentó cifras basales por encima del límite considerado como normal, y por lo tanto también fueron excluidos. La población objeto de estudio fue de 400 pacientes.

\section{Resultados}

\section{Incidencia acumulada de insuficiencia renal aguda}

La incidencia de IRA por criterio RIFLE fue 10,3\% (IC95\% 8,7-11,8) (41/400 pacientes). Utilizando el 


\begin{tabular}{|c|c|c|c|}
\hline Variable & $\operatorname{IR} A(n=41)$ & NoIRA $(n=359)$ & Valorp \\
\hline Sexo femenino & $10(24,4 \%)$ & $119(33,1 \%)$ & 0,26 \\
\hline Edad (años) & $68,5( \pm 9,25)$ & $65,4( \pm 10,1)$ & 0,0612 \\
\hline HTA & $33(80,5 \%)$ & $265(73,8 \%)$ & 0,45 \\
\hline DMIR & $2(4,9 \%)$ & $19(5,3 \%)$ & 1,0 \\
\hline DMNIR & $9(22,0 \%)$ & $89(24,8 \%)$ & 0,84 \\
\hline Dislipemia & $24(58,5 \%)$ & $170(47,4 \%)$ & 0,189 \\
\hline Tabaquismo & $5(12,2 \%)$ & $95(26,5 \%)$ & 0,056 \\
\hline Hiperuricemia & $1(2,4 \%)$ & $13(3,6 \%)$ & 1,0 \\
\hline FAC & $1(2,4 \%)$ & $16(4,5 \%)$ & 1,0 \\
\hline Obesidad & $5(12,2 \%)$ & $44(12,3 \%)$ & 1,0 \\
\hline FEVI (\%) & $54( \pm 12,7)$ & $55( \pm 11,7)$ & 0,608 \\
\hline EuroSCORE & $2,46( \pm 2,83)$ & $1,77( \pm 1,56)$ & 0,016 \\
\hline
\end{tabular}

criterio STS (creatininemia $\geq 2 \mathrm{mg} / \mathrm{dl}$ ) se reduce a 4,0\% (IC95\% 3,0-5,0) (16/400 pacientes).

\section{Gravedad de la insuficiencia renal aguda}

Utilizando la clasificación RIFLE se definió la IRA en tres estadios según el aumento de creatinina sérica: 1 - riesgo (aumento de creatinina $>1,5$ a 2 veces valor basal), 2 - injuria (aumento $>2$ a 3 veces valor basal) y 3 - falla (aumento $>3$ veces). En nuestro grupo, de los pacientes con IRA, 70,7\% (29/41 pacientes) se presentó en estadio 1, 22,0\% (9/41) en estadio 2 y 7,3\% (3/41) en estadio 3.

\section{Mortalidad quirúrgica}

Considerando la definición de STS de mortalidad quirúrgica, se produjeron 15 fallecimientos $(3,8 \%)$. Considerando los pacientes sin IRA, la mortalidad quirúrgica es 1,9\% (7/359 pacientes), mientras que alcanza a $19,5 \%$ (8/41) en los portadores de IRA por criterio RIFLE. Si se utiliza el criterio de IRA de STS, la mortalidad quirúrgica en el grupo sin IRA es $3,1 \%$ (12/384 pacientes) y $18,8 \%$ (3/16) en los que presentan IRA. La asociación entre mortalidad e IRA es significativa usando el criterio RIFLE (OR 12,2 IC95\%: 4,2-35,7 p<0,0001) y STS (OR 7,2 IC95\%:1,8-28,5 $\mathrm{p}=0,0052$ ).

Entre los fallecidos portadores de IRA, 75,0\% (6/8 pacientes) se presentó en estadios RIFLE 2 y 3 (injuria y falla respectivamente).

\section{Variables preoperatorias}

Como en la mayoría de los estudios existe franco predominio del sexo masculino. No se observaron diferencias significativas entre los grupos con respecto a la edad, FEVI y factores de riesgo cardiovascular. Sin embargo, el EuroSCORE es significativamente más alto en los individuos con IRA (tabla 2).

\section{Variables perioperatorias}

Cuando se consideran las variables perioperatorias en el análisis univariado se demuestra una asociación entre la presencia de IRA y la realización de cirugía combinada (sustitución valvular y CRM, doble sustitución valvular, sustitución de aorta ascendente y de válvula), el uso de CEC y clampeo aórtico. Los tiempos de CEC y clampeo aórtico son significativamente más prolongados en los pacientes con IRA. La CRM presentó una asociación inversa con IRA (tabla 3).

Sin embargo, en el análisis multivariado, solo la CRM aislada resultó un predictor negativo de IRA (OR 0,4 IC95\% 0,1-1,0 p=0,047), mientras que la cirugía combinada y el uso de CEC no alcanzaron la significación estadística (OR 1,5 IC95\% 0,6-3,6 p=0,39) y (OR 1,3 IC95\% 0,5-3,4 p=0,63 respectivamente).

\section{Insuficiencia renal aguda y su relación con mortalidad quirúrgica}

En el análisis univariado de la mortalidad quirúrgica los pacientes fallecidos eran significativamente más 
Tabla 3. Variables perioperatorias en ambos grupos. Análisis univariado.

\begin{tabular}{lccc}
\hline Variable & $\operatorname{IR} A(n=41)$ & No IRA $(n=359)$ & Valor $p$ \\
\hline CRM & $17(41,5 \%)$ & $263(73,3 \%)$ & 0,0002 \\
Sustitución valvular & $10(24,4 \%)$ & $47(13,1 \%)$ & 0,059 \\
Sustitución aorta ascendente & $2(4,9 \%)$ & $5(1,4 \%)$ & 0,19 \\
Combinada & $12(29,3 \%)$ & $39(10,8 \%)$ & 0,009 \\
CEC & $30(73 \%)$ & $178(49,6 \%)$ & 0,006 \\
Tiempo CEC (min) & $103( \pm 41,6)$ & $94( \pm 21,2)$ & 0,02 \\
Clampeo aórtico & $24(58,5 \%)$ & $93(25,9 \%)$ & $<0,0001$ \\
Tiempo de clampeo (min) & $85( \pm 27,9)$ & $75,9( \pm 23,6)$ & 0,023 \\
Endocarditis & $3(7,3 \%)$ & $6(1,7 \%)$ & 0,054 \\
BIAC & $3(7,3 \%)$ & $18(5,0 \%)$ & 0,46 \\
Inotrópicos $>48 \mathrm{~h}$ & $10(24,4 \%)$ & $53(14,8 \%)$ & 0,11 \\
\hline CRM: cirugía de revascularización miocárdica; CEC: circulación extracorpórea; BIAC: balón de contrapulsación intraaórtico.
\end{tabular}

Tabla 4. Mortalidad quirúrgica. Variables pre e intraoperatorias. Análisis univariado.

\begin{tabular}{lccc}
\hline Variable & Fallecidos $(n=15)$ & Vivos $(n=385)$ & Valor $p$ \\
\hline Sexo femenino & $6(40,0 \%)$ & $123(31,9 \%)$ & 0,5 \\
Edad (años) & $70,6( \pm 11,7)$ & $65,5( \pm 9,9)$ & 0,036 \\
HTA & $11(73,3 \%)$ & $287(74,5 \%)$ & 0,92 \\
Dislipemia & $8(53,3 \%)$ & $186(48,3 \%)$ & 0,7 \\
IRA & $8(53.3 \%)$ & $33(8,6 \%)$ & $<0,0001$ \\
FEVI (\%) & $52( \pm 14)$ & $55( \pm 11,7)$ & 0,33 \\
EuroSCORE & $3,8( \pm 4)$ & $1,77( \pm 1,54)$ & $<0,0001$ \\
CEC & $10(66,7 \%)$ & $198(51,4 \%)$ & 0,25 \\
Tiempo CEC (min) & $133( \pm 54,3)$ & $96( \pm 27,5)$ & $<0,0001$ \\
Clampeo aórtico & $9(60,0 \%)$ & $108(28,0 \%)$ & 0,012 \\
Tiempo de clampeo (min) & $90,8( \pm 36)$ & $76,7( \pm 23,3)$ & 0,025 \\
\hline HTA: hipertensión arterial; IRA: insuficiencia renal aguda; FEVI: fracción de eyección del ventrículo izquierdo; CEC: circulación \\
extracorpórea.
\end{tabular}

añosos y con EuroSCORE más elevado. La presencia de IRA, el uso de CEC y clampeo aórtico se asociaron a mortalidad. También se observó mayor duración de la CEC y del clampeo aórtico en los fallecidos (tabla 4).

En la regresión logística binaria solo la presencia de IRA resulta predictor independiente de mortalidad (OR 9,6 IC95\%:3,1-29,6 p=0,0000).

\section{Discusión}

La incidencia acumulada de IRA por criterio RIFLE en este estudio fue 10,3\% (IC95\% 8,7-11,8), mayor a la reportada históricamente en el servicio:
8,9\% (IC95\%:8,3-9,5\%) en el que se utilizó el criterio STS. Cuando aplicamos a la población estudiada el criterio STS, observamos que la incidencia se reduce a menos de la mitad: 4\% (IC95\% 3,0-4,98). Esto implica que el criterio STS subestima la insuficiencia renal. Los factores asociados a la IRA son el EuroSCORE más elevado, los procedimientos combinados, el uso de CEC y clampeo aórtico. La CRM se asoció con una incidencia reducida de IRA y aparece como único predictor independiente en la regresión logística. Se encontró una asociación de mortalidad con las siguientes variables: edad mayor, tiempo de CEC y clampeo aórtico mayores y 
EuroSCORE más elevado. Los pacientes que presentaron IRA tuvieron significativamente mayor mortalidad quirúrgica, constituyendo la IRA un predictor independiente en la regresión logística.

La IRA en el POCC se asocia con sustancial morbimortalidad, independiente de otros factores ${ }^{(8)}$. Incluso aumentos modestos de la creatinina sérica basal se han vinculado a un incremento significativo de la mortalidad a 30 días $^{(6)}$. La incidencia de IRA en este trabajo se encuentra dentro de los valores aportados por la bibliografía, haciendo la salvedad de su amplia variación de acuerdo a los criterios considerados. Pudo comprobarse que el criterio histórico STS subestima a los portadores de IRA, dado que el punto de corte utilizado no toma en consideración los valores basales. El criterio RIFLE fue publicado por el Acute Dialysis Quality Initiative group en $2004^{(17)}$ con la finalidad de estandarizar las definiciones de IRA. Posteriormente, en 2007, surgió la clasificación AKIN y ambas han probado su utilidad en determinar la severidad del daño renal en pacientes críticos ${ }^{(22,23)}$. Por otra parte, la clasificación AKIN no mejoró la sensibilidad ni la habilidad para predecir resultados en pacientes graves. El número de trabajos publicados con el criterio RIFLE sobrepasa los 150, y en algunas revisiones el número de pacientes es cercano a $70.000^{(24)}$.

Con respecto a las variables asociadas a IRA, el tipo de cirugía, específicamente los procedimientos combinados se asociaron con un aumento significativo de su incidencia. Este resultado está de acuerdo con la evidencia internacional, siendo los procedimientos aislados (cirugía coronaria y valvular) los que tienen menor incidencia $(2,5 \%$ y $2,8 \%$, respectivamente), mientras que los procedimientos combinados alcanzan el 4,8\%(11). Los procedimientos valvulares mostraron una tendencia al aumento que no alcanza significación estadística.

El uso de CEC y clampeo aórtico se asociaron a la presencia de IRA, observándose tiempos quirúrgicos mayores en este grupo. Se atribuye un papel determinante en la generación del insulto renal a la hemólisis producida por el uso de rodillos y a la succión del reservorio de cardiotomía, así como a la liberación de hemoglobina y radicales libres. También la hemodilución que se genera durante la CEC sería responsable de isquemia renal medular ${ }^{(9,10,12)}$. La canulación y el clampeo aórtico se asocian con incremento de fenómenos ateroembólicos renales que exacerban la isquemia e inducen inflamación ${ }^{(26)}$. Sin embargo, el efecto protector de la cirugía sin CEC sobre la incidencia de IRA sigue siendo controvertido. En varios estudios recientes, predominantemente de grandes dimensiones, la cirugía sin CEC se asoció con un menor riesgo de desarrollar IRA, mientras que algunos estudios más pequeños no confirmaron un efecto beneficioso. Un metaanálisis reciente tampoco pudo resolver este problema, ya que demostró una reducción de la presencia de IRA mediante la técnica sin CEC en varios estudios observacionales ajustados al riesgo en grandes cohortes de pacientes, pero no en un número menor de estudios controlados aleatorios con un limitado número de pacientes ${ }^{(27)}$. Existen dos posibles explicaciones para estos resultados contradictorios. En primer lugar, los estudios previos aplicaron definiciones ampliamente variadas y no estandarizadas de IRA, que van desde pequeños aumentos de la creatinina sérica hasta el requerimiento de terapia de sustitución renal. En segundo lugar, los estudios previos analizaron un número limitado de factores de riesgo de lesión renal aguda y no se consideraron los factores de riesgo posoperatorios potentes ${ }^{(28)}$.

Cuando se realizó la regresión logística, no pudo demostrarse que estas variables sean predictoras de IRA. Solo la CRM aparece vinculada a menor incidencia de IRA. Una explicación a este resultado podría atribuirse a la no utilización de CEC ni de clampeo aórtico.

Otras variables consideradas habitualmente como factores de riesgo de aparición de IRA como la edad, el sexo femenino, la DM, la FEVI y la utilización de BIAC no pudo demostrarse que lo fueran en nuestro estudio(6,9).

El pronóstico de los pacientes que sufren IRA en el POCC es pobre, siendo la mortalidad entre $10 \%$ a $80 \%$ mayor que los controles. La infección se considera la causa principal de los fallecimientos $(40 \%)^{(25,27)}$. Acorde con la evidencia, en nuestro trabajo, $50 \%$ de los pacientes fallecidos cumplía criterios de IRA y se identificó como predictor de mortalidad.

En el estudio de Hobson y colaboradores ${ }^{(16)}$ se analizaron retrospectivamente 2.973 pacientes sin historia de ERC sometidos a cirugía cardíaca e igual que en el presente estudio se utilizó la clasificación RIFLE para definir IRA. La sobrevida fue menor en los pacientes que presentaron deterioro de la función renal. Cuanto mayor es el deterioro de la función, mayor es la mortalidad, como se comprueba en nuestro trabajo.

\section{Limitaciones}

Se trata de un estudio unicéntrico donde se usó de forma aislada la creatinina sérica como marcador de disfunción renal. La utilización de fármacos con efecto renal no fue regulada, aunque al presente ninguna estrategia farmacológica-dopamina, antagonistas de los canales cálcicos, diuréticos de asa, péptidos natriuréticos, IECA- ha demostrado de 
forma concluyente prevenir la disfunción renal luego de cirugía cardíaca ${ }^{(9,28)}$. El período de observación fue solamente de 48 horas, lo que implica que eventuales pacientes con manifestación tardía de deterioro renal (hecho verificado en algunos trabajos $\left.{ }^{(30)}\right)$ no pudieron ser detectados. Del mismo modo, desconocemos cuál fue la cinética de la función renal a posterioriy si los pacientes normalizaron las cifras de creatininemia al alta.

\section{Bibliografía}

1. Lema G, Canessa R, Urzúa J, Jalil R, Morán S, Carvajal C, et al. Función renal en cirugía cardíaca con circulación extracorpórea: Pacientes valvulares y coronarios. Rev Méd Chile 2008; 136(4):459-66.

2. Chertow GM, Burdick E, Honour M, Bonventre JV, Bates DW. Acute kidney injury, mortality, length of stay, and costs in hospitalized patients. J Am Soc Nephrol 2005; 16(11):3365-70.

3. Mangano CM, Diamondstone LS, Ramsay JG, Aggarwal A, Herskowitz A, Mangano DT. Renal dysfunction after myocardial revascularization: risk factors, adverse outcomes, and hospital resource utilization. The Multicenter Study of Perioperative Ischemia Research Group. Ann Intern Med 1998; 128(3):194-203.

4. Lassnigg A, Schmidlin D, Mouhieddine M, Bachmann LM, Druml W, Bauer P, et al. Minimal changes of serum creatinine predict prognosis in patients after cardiothoracic surgery: a prospective cohort study. J Am Soc Nephrol 2004; 15(6):1597-605.

5. Hudson C, Hudson J, Swaminathan M, Shaw A, Stafford-Smith M, Patel UD. Emerging concepts in acute kidney injury following cardiac surgery. Semin Cardiothorac Vasc Anesth 2008; 12(4):320-30.

6. Gude D, Jha R. Acute kidney injury following cardiac surgery. Ann Card Anaesth 2012; 15(4):279-86.

7. Conlon PJ, Stafford-Smith M, White WD, Newman MF, King S, Winn MP, et al. Acute renal failure following cardiac surgery. Nephrol Dial Transplant 1999; 14(5):1158-62.

8. Chertow GM, Levy EM, Hammermeister KE, Grover F, Daley J. Independent association between acute renal failure and mortality following cardiac surgery. Am J Med 1998; 104(4):343-8.

9. Rosner MH, Okusa MD. Acute kidney injury associated with cardiac surgery. Clin J Am Soc Nephrol 2006; 1(1):19-32.

10. Palomba H, de Castro I, Neto AL, Lage S, Yu L. Acute kidney injury prediction following elective cardiac surgery: AKICS Score. Kidney Int 2007; 72(5): 624-31.

11. Grayson AD, Khater M, Jackson M, Fox MA.
Valvular heart operation is an independent risk factor for acute renal failure. Ann Thorac Surg 2003; 75(6):1829-35.

12. Boldt J, Brenner T, Lehmann A, Suttner SW, Kumle B, Isgro F. Is kidney function altered by the duration of cardiopulmonary bypass? Ann Thorac Surg 2003; 75(3):906-12.

13. Di Mauro M, Gagliardi M, Iacò AL, Contini M, Bivona A, Bosco P, et al. Does off-pump coronary surgery reduce postoperative acute renal failure? The importance of preoperative renal function. Ann Thorac Surg 2007; 84(5):1496-502.

14. Bucerius J, Gummert JF, Walther T, Schmitt DV, Doll N, Falk V, et al. On-pump versus off-pump coronary artery bypass grafting: impact on postoperative renal failure requiring renal replacement therapy. Ann Thorac Surg 2004; 77(4):1250-6.

15. Karkouti K, Wijeysundera DN, Yau TM, Callum JL, Cheng DC, Crowther M, et al. Acute kidney injury after cardiac surgery: focus on modifiable risk factors. Circulation 2009; 119(4):495-502.

16. Hobson CE, Yavas S, Segal MS, Schold JD, Tribble CG, Layon AJ, et al. Acute kidney injury is associated with increased long-term mortality after cardiothoracic surgery. Circulation 2009; 119(18):2444-53.

17. Bellomo R, Ronco C, Kellum JA, Mehta RL, Palevsky P. Acute renal failure: definition, outcome measures, animal models, fluid therapy and information technology needs: the Second International Consensus Conference of the Acute Dialysis Quality Initiative (ADQI) Group. Crit Care 2004; 8(4): R204-12.

18. Toffaletti JG. Improving the clinical value of estimating glomerular filtration rate by reporting all values: a contrarian viewpoint. Nephron Clin Pract 2010; 115(3):c177-81.

19. Owen JA, Iggo B, Scandrett FJ, Stewart CP. The determination of creatinine in plasma or serum, and in urine; a critical examination. Biochem J 1954; 58(3):426-37.

20. Lagny MG, Jouret F, Koch JN, Blaffart F, Donneau AF, Albert A, et al. Incidence and outcomes of acute kidney injury after cardiac surgery using either criteria of the RIFLE classification. BMC Nephrol 2015; 16:1-9.

21. Jacobs JP, Mavroudis C, Jacobs ML, Maruszewski B, Tchervenkov CI, Lacour-Gayet FG, et al. What is operative mortality? Defining death in a surgical registry database: a report of the STS Congenital Database Taskforce and the Joint EACTS-STS Congenital Database Committee. Ann Thorac Surg 2006; 81(5):1937-41.

22. Khan S, Kabir J. Perioperative acute kidney injury in cardiac surgery. Bangladesh Heart J 2015; 30(2): 78-86. 
23. Lin CY, Chen YC. Acute kidney injury classification: AKIN and RIFLE criteria in critical patients. World J Crit Care Med 2012; 1(2):40-5.

24. Candela Toha AM, Recio Vázquez M, Delgado Montero A, del Rey JM, Muriel A, Liaño F, et al. El cálculo de la creatinina sérica basal sobreestima el diagnóstico de alteración renal aguda en pacientes operados de cirugía cardíaca. Nefrología (Madr.) 2012; 32(1):53-8.

25. Thakar CV, Worley S, Arrigain S, Yared JP, Paganini EP. Influence of renal dysfunction on mortality after cardiac surgery: modifying effect of preoperative renal function. Kidney Int 2005; 67(3):1112-9.

26. O’Neal JB, Shaw AD, Billings FT 4th. Acute kidney injury following cardiac surgery: current understanding and future directions. Crit Care 2016; 20(1):187.

27. Lok CE, Austin PC, Wang H, Tu JV. Impact of renal insufficiency on short and long-term outcomes after cardiac surgery. Am Heart J 2004; 148(3):430-8.

28. Kolh P. Renal insufficiency after cardiac surgery: a challenging clinical problem. Eur Heart J 2009; 30(15):1824-7.
29. Wijeysundera DN, Beattie WS, Djaiani G, Rao V, Borger MA, Karkouti K, et al. Off-pump coronary artery surgery for reducing mortality and morbidity: meta-analysis of randomized and observational studies. J Am Coll Cardiol 2005; 46(5):872-82.

30. Bastin AJ, Ostermann M, Slack AJ, Diller GP, Finney SJ, Evans TW. Acute kidney injury after cardiac surgery according to Risk/Injury/Failure/Loss/End-stage, Acute Kidney Injury Network, and Kidney Disease: Improving Global Outcomes classifications. J Crit Care 2013; 28(4):389-96. doi:10.1016/j.jcrc.2012.12.008.

31. Massoudy $\mathbf{P}$, Wagner S, Thielmann M, Herold U, Kottenberg-Assenmacher E, Marggraf G, et al. Coronary artery bypass surgery and acute kidney injury-impact of the off-pump technique. Nephrol Dial Transplant 2008; 23(9):2853-60.

32. Roh GU, Lee JW, Nam SB, Lee J, Choi JR, Shim YH. Incidence and risk factors of acute kidney injury after thoracic aortic surgery for acute dissection. Ann Thorac Surg 2012; 94(3):766-71. 